\title{
A New Pathway towards Retirement Preparation: Integration of Holistic Life Planning
}

\author{
Dayang Kartini Abang lbrahim \\ Nor Wahiza Abdul Wahat (Phd) \\ Faculty of Educational Studies, \\ Universiti Putra Malaysia, Malaysia \\ Email: vikaqina@yahoo.co.uk
}

Abstract

This is a concept paper to review the needs of holistic life planning as a new pathway towards retirement preparation. It is based on the review of literature on the importance of this knowledge to fulfil individual successful planning and preparation for retirement. It includes the review on a planning process theory by Friedman and Scholnick (1997) which provides the basis for generation of retirement preparation. Meanwhile Hansen's Integrative Live Planning (ILP) theory is embedded within the theoretical framework to help better understanding on the domains of planning and preparation for retirement. The purpose is to develop a theoretical model for measuring employees' retirement preparation in government organizations. The primary problem in retirement preparation is the lack of theory to show the development of retirement preparation process. Although prior research has theories explained on retirement thoughts, behaviors, attitudes or goals undertaken to fulfill the retirement preparation, none of it measures applied in the studies were established to directly test the theories according to the process and development of retirement. According to the process theory, individuals acquire an understanding of the problem, goals will be set in making a decision to start preparing and finally behaviors is considered necessary to fulfil the goals (Noone et al., 2009). This raises intriguing questions: What factors influence retirement preparation? Which stages in retirement planning and preparation require intervention and support? (Noone et al., 2009) Which domain in life is significant to retirement preparation? The theory is discussed throughout the paper in the context of the needs of retirement preparation. This paper would be useful for policy makers, practitioners and employees in considering their own retirement preparation for future wellbeing.

Keywords: Retirement Preparation, Retirement Planning, Hansen's Integrative Life Planning (ILP), A process theory of planning, Retirement Domains. 